\title{
LA-UR- $98=4856$
}

Approved for public release; distribution is unlimited.

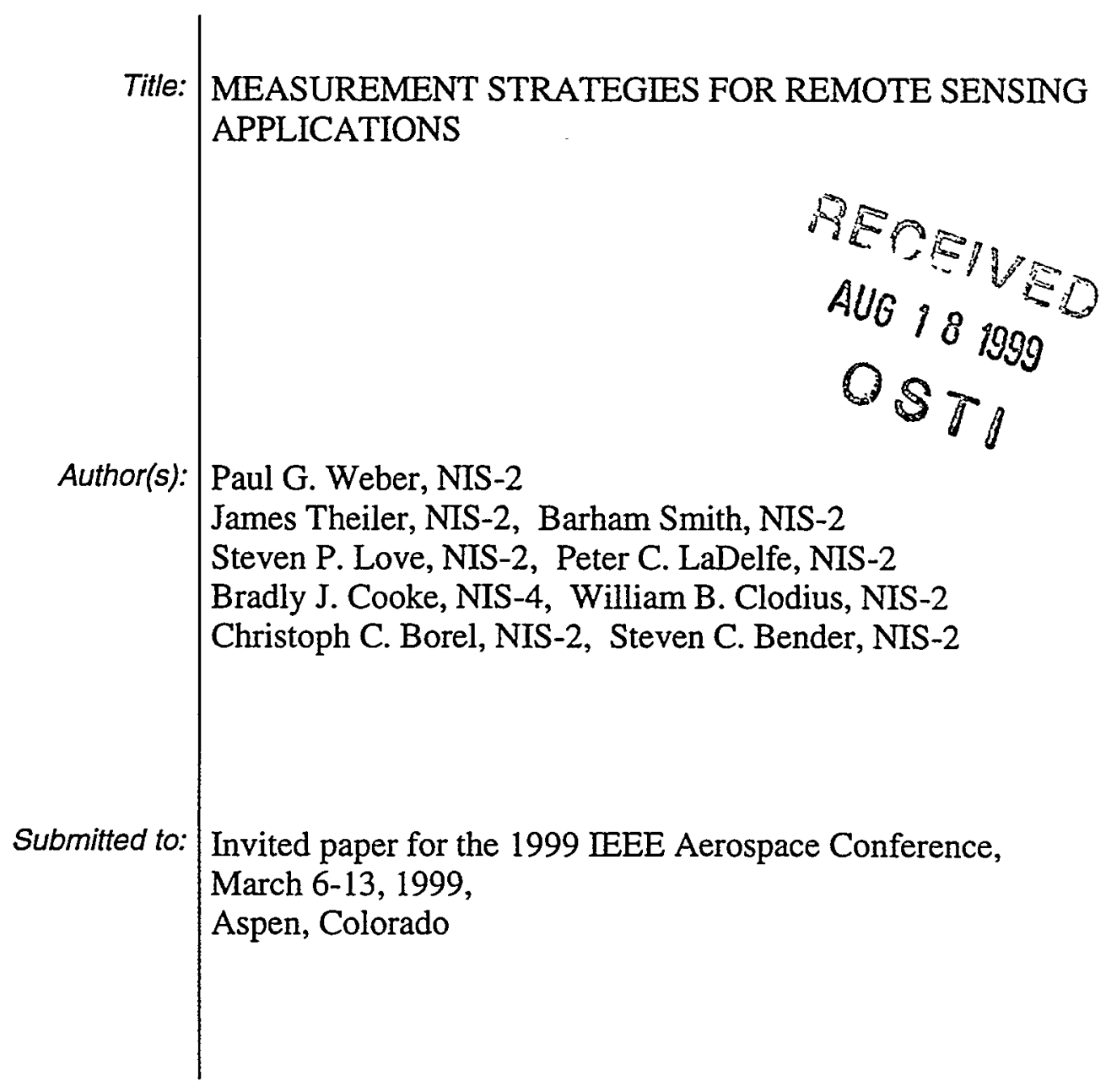

\section{Los Alamos NATIONAL LABORATORY}

Los Alamos National Laboratory, an affirmative action/equal opportunity employer, is operated by the University of California for the U.S. Department of Energy under contract W-7405-ENG-36. By acceptance of this article, the publisher recognizes that the U.S. Government retains a nonexclusive, royalty-free license to publish or reproduce the published form of this contribution, or to allow others to do so, for U.S. Government purposes. Los Alamos National Laboratory requests that the publisher identify this article as work performed under the auspices of the U.S. Department of Energy. Los Alamos National Laboratory strongly supports academic freedom and a researcher's right to publish; as an institution, however, the Laboratory does not endorse the viewpoint of a publication or guarantee its technical correctness. 


\section{DISCLAIMER}

This report was prepared as an account of work sponsored by an agency of the United States Government. Neither the United States Government nor any agency thereof, nor any of their employees, make any warranty, express or implied, or assumes any legal liability or responsibility for the accuracy, completeness, or usefulness of any information, apparatus, product, or process disclosed, or represents that its use would not infringe privately owned rights. Reference herein to any specific commercial product, process, or service by trade name, trademark, manufacturer, or otherwise does not necessarily constitute or imply its endorsement, recommendation, or favoring by the United States Government or any agency thereof. The views and opinions of authors expressed herein do not necessarily state or reflect those of the United States Government or any agency thereof. 


\section{DISCLAIMER}

Portions of this document may be illegible in electronic image products. Images are produced from the best available original document. 


\title{
Measurement Strategies for Remote Sensing Applications.
}

\author{
Paul G. Weber, James Theiler, Barham W. Smith, Steven P. Love, Peter C. LaDelfe, Bradly J. Cooke, William B. Clodius, \\ Christoph C. Borel, and Steven C. Bender. \\ Space and Remote Sensing Sciences Group, Los Alamos National Laboratory. \\ Correspondence to: Paul G. Weber, Group Leader, NIS-2, D436, Los Alamos National Laboratory, Los Alamos, NM 87545, \\ USA. (505)667-5776, pweber@lanl.gov
}

\begin{abstract}
Remote sensing has grown to encompass many instruments and observations, with concomitant data from a huge number of targets. As evidenced by the impressive growth in the number of published papers and presentations in this field, there is a great deal of interest in applying these capabilities. The true challenge is to transition from directly observed data sets to obtaining meaningful and robust information about remotely sensed targets. We use physicsbased end-to-end modeling and analysis techniques as a framework for such a transition. Our technique starts with quantified observables and signatures of a target. The signatures are propagated through representative atmospheres to realistically modeled sensors. Simulated data are then propagated through analysis routines, yielding measurements that are directly compared to the original target attributes. We use this approach to develop measurement strategies which ensure that our efforts provide a balanced approach to obtaining substantive information on our targets.
\end{abstract}

\section{INTRODUCTION}

Deploying sophisticated remote sensing systems in space, or on aircraft, provides us with information on activities, events, and trends around the world. A large number of sensors has been deployed to observe the Earth and its environment (see [1], [2], [3] for summary information). Examples of current data and interpretations from these instruments abound (see the annual proceedings of the IEEE Aerospace, IGARSS, SPIE, and COSPAR conferences, and journals including IEEE Transactions on Remote Sensing, Remote Sensing of the Environment, and others). Organizations investing in remote sensing include governmental and industrial entities. We see a plethora of applications, instruments, data, and interpretations!
Our goal, however, is to obtain robust and meaningful information from our data. Thus we must ensure that our measurement strategy is well understood before we commit to particular system choices. These choices may include the types of instruments, additional data needed from other sources, accuracy and precision of all data, spatial and temporal sampling, etc. That is, we need to understand not just the sensor, but the sensor in the context of the target observables, signatures, intervening atmosphere, platform, optics, electronics, data system, and analysis packages. All of these items are interacting elements of a measurement strategy, and also drive requirements that need to be balanced against system cost [4]. An appropriate set of measurement strategies permits sensible quantitative trades of technological choices against the true bottom line. Our ultimate measure of success is: "How well will our measurement strategy provide cost-effective answers to specific questions posed by our customers?" We should understand all the elements that affect the quality of the answer: we may well be able to reduce costs for items where changes do not significantly impact the bottom line answers.

Customer questions may include "What is this facility?" and "Do we see evidence of global climate change?" We can address such questions phenomenologically, or by working through a complete, closed-loop, physics-based model of the measurement system. We define "physics-based" as using information from physical understanding (as represented by equations) in contrast to using test results or scaling approximations. We emphasize here the benefits of basing the models in physics, since this generates confidence in the ability, for example, to extend our validated results from previous applications to new regimes. However, full confidence can only be achieved from a physics-based system that incorporates all of the important attributes of the whole measurement strategy. 


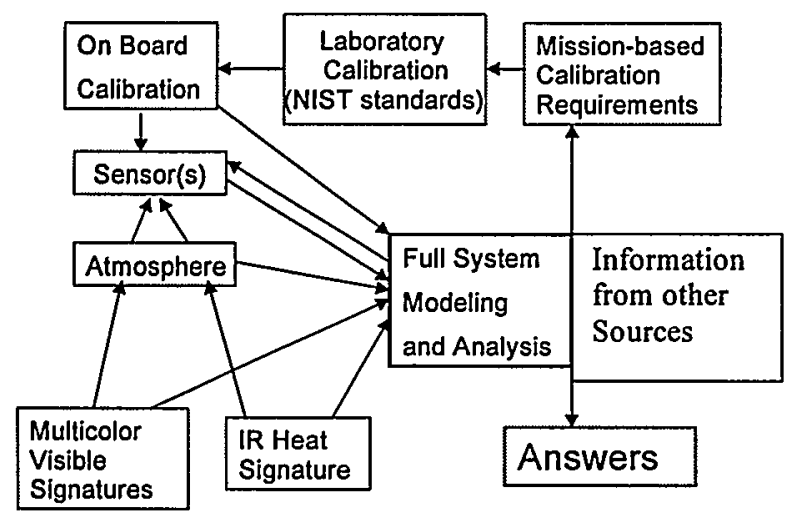

Figure 1: Design of measurement strategies

A greatly simplified and rather generic flow diagram for developing a measurement strategy is shown in Figure 1. The true bottom line is at the lower right: answers to questions posed by our customers. The central focus for the strategy is the Full System Modeling and Analysis box, and we attempt to balance the system elements by examining effects of these elements on the final answers. Let us follow a sequence through an actual measurement strategy: We translate the customer questions into a series of pertinent local observables and associated signatures, which we then propagate through the atmosphere to a proposed sensor system. The modeled sensor takes the signatures (e.g. IR spectral radiances) and converts them to digital data; we include here relevant distortions due to such factors as hardware imperfections, platform motion, jitter, electronic noise, and detector non-linearity. We then apply analysis tools to these ersatz data to retrieve the best rendition of the original signatures. Comparing the retrieved information to the known original scene allows us to judge the efficacy of the full measurement strategy. Use of such a physics-based End-to-End Model (EEM) allows the establishment of system attributes in a balanced fashion, recognizing limitations and costs of hardware, calibration systems, analysis routines, and natural variability within our target ensembles and intervening sources of distortion such as the atmosphere. After the measurement strategy is refined and balanced, we substitute "as-built" hardware attributes into the model, eventually replacing the model with hardware and maintaining (or enhancing) the analysis segment. As just one benefit of the EEMs, the modeling defines the desired attributes of sensor calibration; combining this with our knowledge of what is practical and affordable allows us to build and implement the most appropriate ground-based and on-board calibration systems. Specifying "the most accurate" calibration system for each of these systems that we have implemented would be wasteful of resources.

We have applied this philosophy to a space-borne multispectral imaging system, hyperspectral remote sensing, and a Lidar system. In this paper we will address just one of these applications: the Multispectral Thermal Imager funded by the Department of Energy (DOE). However, the principles elucidated here have applicability to a wide spectrum of remote sensing applications. This paper illustrates some of the benefits of our physics-based approach.

\section{APPLICATION TO IMAGING SPECTROSCOPY}

We use imaging spectroscopy in the visible and infrared spectral regions to illustrate our approach. This is an area of considerable interest, with applications to atmospheric sciences, hydrology, oceanography, ecology, geology [5], to environmental sciences [6], and to a variety of national security applications [7]. Our specific focus here will be on the accurate measurements of signatures including materials identification, vegetation stress, change detection, and of Sea Surface Temperature (SST) and Land Surface Temperature (LST) from airborne and space-based instruments. In the interest of brevity we will focus here on the temperature retrievals, though the same principles apply to accurate or precise retrievals of other signatures: namely, one needs to understand the full measurement strategy to implement defensible choices toward a self-consistent and cost-effective systems approach.

A long-term database from weather satellites exists on low spatial resolution SST [8] and for LST $[9,10]$. Comparison of these remotely sensed data to ground truth indicate reasonable agreement for known target emissivities under favorable weather conditions. At least two programs are now targeting a number of multispectral signatures including accurate temperature measurements at high spatial resolutions: the MODIS and ASTER instruments on NASA's Mission to Planet Earth [11], and the DOE's Multispectral Thermal Imager (MTI) satellite [12]. The goals of the NASA instruments are to provide full global coverage at moderate resolution; the DOE mission involves local measurements at high spatial resolution. Each of these programs has invested in modeling and analysis, and in sophisticated calibrations, and each will conduct comparisons of remotely sensed data to ground truth when the satellites fly in late 1999 to validate system performance $[13,14]$. Here we discuss the measurement strategy for MTI.

We begin with the attributes of the target to be examined: typical interests for the DOE include large industrial facilities, such as power plants, with associated infrastructure and its surroundings. These targets have feature sizes of many meters, which sets our spatial resolution. To study nearby environmental impacts, we wish to cover a swath width on the order of ten- (10) km. Thus our end-to-end models begin with thermal maps, generated from airborne data, from 3-D modeling of cooling systems [15], from thermal models (Rochester Institute of Technology's DIRSIG codes) or other sources. This is the truth basis for our modeling: we know the emissivities (as a function of wavelength) and temperatures in the scene. 
The emissivity of water is close to unity, but still varies as a function of surface roughness [16] including possible shadowing effects [17]. Similarly, there is a finite polarization effect to be considered [18]. The models used here have been cross-checked with data [19] to ensure fidelity.

Propagation through the atmosphere leads to both a distortion of the signature due to absorption and scattering, as well as the addition of path radiance from the atmosphere itself $[20,21]$. Atmospheric gases such as $\mathrm{CO}_{2}, \mathrm{O}_{3}$, and CFCs are known to vary over larger spatial scales, and we assume them to be uniformly distributed over our swath. The major atmospheric variables are water vapor, clouds, and aerosols; their effects on SST and LST retrievals from weather satellites have been well-documented [8], [22] and [23]. We address each one of these major atmospheric variables in our system.

Atmospheric water vapor over the oceans is measured well by the Special Sensor Microwave Imager (SSM/I) instruments on the polar orbiting satellites of the Defense Meteorological Satellite Program (DMSP). The rms. accuracies of wind speed, water vapor, and cloud water are $1 \mathrm{~m} / \mathrm{s}, 1 \mathrm{~mm}$, and $0.03 \mathrm{~mm}$ respectively (IEEE newsletter 9/1997). However, the spatial grid size is 0.25 degrees, and spatial coverage is only four times per day. Observations of spatial and temporal variability of the atmospheric water vapor indicate changes on much faster scales. Fortunately one can employ a relatively simple spectroscopic technique to measure column water vapor to an accuracy of five percent with high spatial resolution[25], thus ameliorating the effects of this variable quantity.

Several techniques have been developed to detect visible clouds using such characteristics as relatively large spatial extent, high albedo, lack of correlation with land surface features and low temperatures [26] and [27]. Cirrus clouds, or for atmospheric aerosols, we invoke the detection method of Gao, Goetz and Wiscombe [28] using the strong water vapor absorption feature at 1.375 microns. Gao, Davis and Kaufman [29] have described a correction method for the influence of Cirrus clouds on visible and near-infrared data from the airborne AVIRIS sensor.

Thus, we can measure the key atmospheric variables at the desired spatial resolutions and contemporaneously with the temperature data. This permits correction of the atmospheric effects in the analysis segments.

Our optical system is modeled by Code V, or, for less stressing applications, it can be approximated by the Point Spread Function (PSF) and the Modulation Transfer Function (MTF). Other attributes of the detector system are modeled in detail as described, for example, by Cooke, et al [30]. Rienstra and Ballard [31] have described the multispectral focal plane assembly. Our goal was to have a minimum number of spectral bands needed to achieve the mission goals -- and the EEM helped us to reach that point.
Modern electronics have excellent properties, and the downlinks from satellites to ground stations have very low error rates. Nevertheless, we face non-linearities and finite digitization levels, including decisions on how many digitization levels are needed in the face of limitations elsewhere in the system. Specific techniques and algorithms are used to achieve these high quality data: we model them as an integral part of the EEM.

These steps bring us to the end of the forward, or modeling, part of the end-to-end model. At this point we have simulated data with crudely defined spectral bands and other attributes, which we can use as input to the analysis algorithms that follow. As real attributes of the hardware are realized, we insert those quantities into the EEM, thus improving fidelity, and testing the adequacy of delivered hardware within the full context of the measurement strategy.

We now add the analysis segment to complete the EEM. We started with relatively simple techniques, and added more sophistication as we recognized the critical aspects of the measurement strategy, and as we learned of the realities of the available and affordable, hardware.

Let us begin by examining the temperature retrievals at night, when we have measurable photon fluxes only in the mid-wave and long-wave infrared spectral regions (MWIR defined here as 3-5 microns and LWIR as 8-14 microns). Based on the common "split-window" technique used on weather satellites, one might settle for one band in the MWIR and two in the LWIR to permit a reasonable fit to the Planck spectrum as modified by the atmosphere. However, as a starting point for a new system, we were not constrained to a specific technique, or to the heritage of specific spectral bands. Thus we considered that we have opportunity to measure not just in a relatively clear part of the spectrum, but also to add bands which will be heavily affected by water vapor and by the variable trace gases. This allows a better characterization of the atmospheric state, and a better correction of atmospheric effects. As an example, water vapor absorption is significant at the short wavelength end of the LWIR band: placing one spectral band on the absorption edge, and an adjacent band in a relatively clear spectral region allows us to obtain some information on the column integrated water vapor content. As another example, one can use two bands in the MWIR: one on each side of the large $\mathrm{CO}_{2}$ absorption feature: the spectrum is relatively clear on the short wavelength side, and quite contaminated on the long side, again permitting an additional measure of atmospheric condition. These considerations gave a first guide to spectral band selection, with a sense that five spectral bands may be enough to provide the needed data, and a sense those additional bands may be redundant. Much detailed modeling then refined the band choices [32] and confirmed our initial estimates of the needed information. 
We applied the same methodology to temperature retrievals by day, with much added information provided by reflected solar radiation. The starting point for these data includes the closely-studied Landsat spectral bands, plus data from airborne multi- and hyperspectral sensors. The added solarreflected data includes information on materials in the scene at high spatial resolution, which permits derivation of emissivities and definition of boundaries between materials. Further, we obtain the column-integrated water content from data around 0.9 to 1.0 microns, and data on contamination of the upper atmosphere from data at 1.375 microns. Not all is positive, however, since the solar spectrum adds significantly to the short wavelength end of the MWIR region, thus contaminating the shortest MWIR band beyond usefulness. Again one iterates from this starting point toward definition of the best choices in spectral bands (see Figure 2, adapted from Clodius, et al [32]). The figure of merit here is to obtain spectral bands with maximum sensitivity for each of the desired data products ("answers" in the nomenclature of Figure 1), and with low sensitivity to contamination by other effects.

The major output of this process is a definition of the spectral bands and a determination of the endemic uncertainties in the system. These uncertainties include the fact that the fidelity of the models is not perfect, and the fact that the hardware elements will each have tolerances and deviations from the desired attributes. Indeed, we can use the models to set the desired tolerances on the hardware in a self-consistent manner. The other outputs are the radiometric accuracies and precisions for the system, as well as the alignment and jitter limits. The latter limits are referred to the specifications for the spacecraft bus: methodologies for balancing the spacecraft and launch vehicle technologies are the subject of a recent study [33] and of an extensive monograph [34].

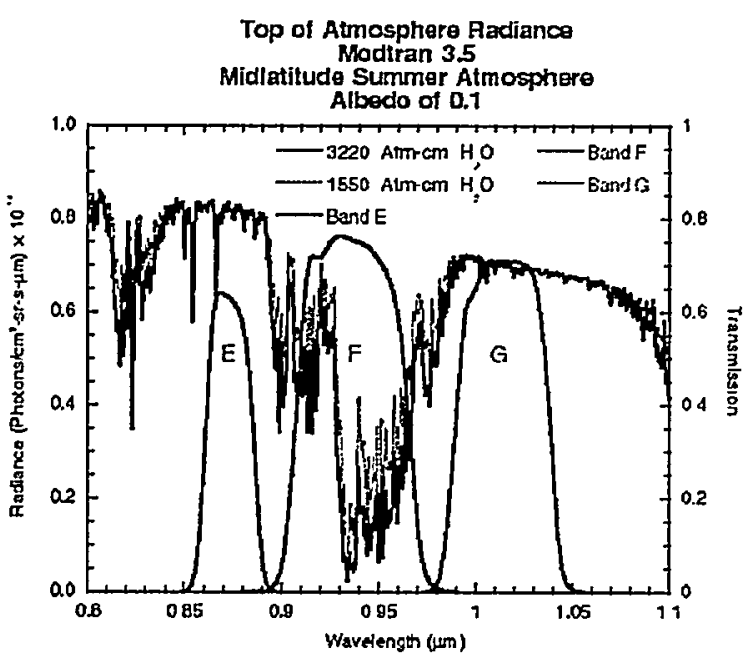

Figure 2: Water vapor effects in the VNIR
In all of this modeling, an effort is made to insert reasonable expectations for achievable hardware performance. Indeed, the end-to-end modeling yields a self-consistent set of specifications, in that one can see the effect of any given variation on the final retrievals. Based on this information, one now iterates with vendors of real hardware, inserting true performance specifications, and ensuring that the required bottom-line performance is maintained. Similarly, in the event that a piece of hardware is delivered and fails to meet specifications, one can assess the effects on overall performance and make solid decisions on whether to accept or reject such parts. As an example of such a trade, our original specifications for the longest wavelength spectral bands extended to 11.5 microns. The supplier pointed out that this value was stressing his technologies, and offered three options: (a) use different stochiometric mixtures of $\mathrm{HgCdTe}$ for longer and shorter wavelengths; (b) use a mixture which optimizes performance in the longest wavelength band, but at a cost of inferior signal-to-noise in the shorter wavelength bands; or (c) shift the upper cutoff wavelength of the longest wavelength band to 10.8 microns. We studied these options quantitatively, and determined that we could, in fact, drop the upper wavelength limit to 10.8 microns with a tolerable effect on the true bottom line. The other options were also studied, but were, in the end, rejected.

Another attribute of the EEM is the ability to test software corrections to specific hardware limitations. As an example in MTI, we originally anticipated a focal plane assembly with fifteen linear arrays covering the full swath width. The reality of focal plane manufacturing indicates limited length of available materials, which led to a focal plane with each array covering just over one-third of the full swath width. The final configuration, shown in Figure 3, has three nominally identical Sensor Chip Assemblies (SCAs) arranged to cover the swath width with some overlap [31]. (As another trade study, we examined the impacts of swath width on number of SCAs, data rate, satellite pointing accuracy, system complexity, cost and performance.) In any case, the configuration with multiple SCAs implies that the data for each spectral band is obtained at multiple times as the satellite traverses the scene.

Re-assembling the data from each spectral band, and reregistering the bands to form a multispectral image is a challenging problem. This applies for a simple push-broom focal plane array with fifteen linear detectors, but gets even more complicated for the three SCAs that we have in hardware. In either case, the satellite will exhibit some jitter during the time that the various spectral bands scan over a scene. The satellite does not have accurate jitter sensors, and the vibration sensors that do exist are located in a place that does not accurately reflect the full impact of vibrations on the imagery. Thus we will have some indicators of spectral vibrational power, but insufficient data to drive a reconstruction algorithm. Therefore, we developed a method, based on the fact that the information in some spectral bands is highly correlated, to reconstruct the jitter 
directly from the multispectral data; this permits us to correctly register each pixel [35]. We were able to show that the desired bottom line performance of the system could be obtained with this different approach to registration.

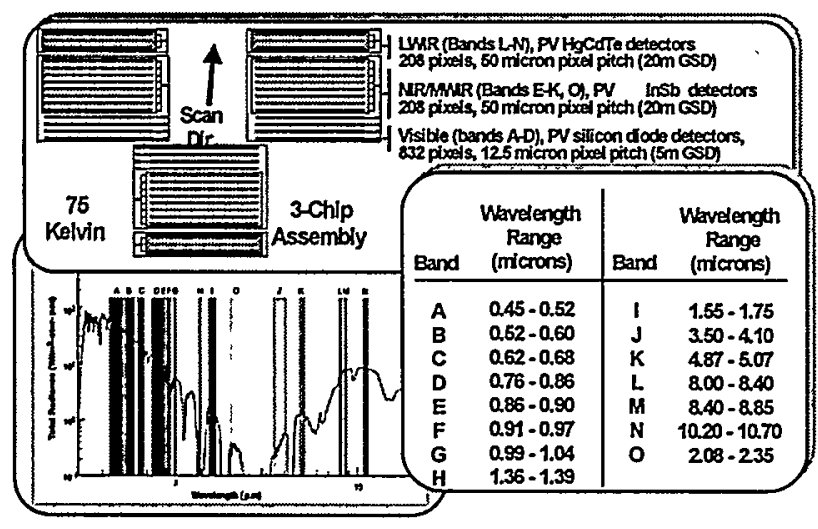

Figure 3: Selection of spectral bands and implementation on the Focal Plane Assembly.

The EEM demonstrated conclusively the importance of correcting for the atmosphere in determining the highest quality retrievals of terrestrial signatures. This validates the incorporation of the atmospheric retrieval bands into the design, but also opens the question of whether we could do better still. This led to the idea of using multiple observations at different observing angles, incorporating the same ground scene with different path lengths through the atmosphere. Of course some caveats apply: the atmosphere must be spherically uniform and unchanged in the time between images, and we need to be concerned about possible changes in emissivity as a function of observing angles, and shadowing. Some data exist on angular emissivity of materials (e.g. the NEF database), and we have computed the effects of angle of observation for water under a variety of weather conditions: these latter results have been successfully compared to shipboard observations [19]. Further information on angular distributions will be forthcoming from analysis of data from the NASA Multiangle Imaging Spectro-Radiometer, MISR [36]. For our MTI application we modeled the effectiveness of multiple looks for different angles, and for different numbers of observations: our trade space study resolved that a nadir look plus one other observation at 45-60 degrees from nadir is most appropriate for our observations. Diminishing returns were noted from adding further look angles, so we restricted ourselves to just two for normal operations. The trade study here compared the costs of slewing the satellite quickly to the benefits of a factor of order two in temperature accuracy; since the satellite was already threeaxis stabilized the incremental costs were manageable and the benefits were very tangible on the bottom line.

We now turn to calibrations. During the initial systems phase, we decided, somewhat arbitrarily, to divide the total error budget equally between the calibration of the hardware, and the uncertainties in modeling and analysis.
As we proceeded to a better understanding, this turned out to be a reasonable choice. (In terms of Figure 1, we are balancing the calibration loop with the modeling/analysis loop.) The calibration segment has several components: establishing laboratory standards, transferring those standards to an on-board calibration system for in-orbit maintenance, and vicarious calibrations checking the performance against ground truth. And again, all of these elements need to be balanced. Again our approach yielded quantitative benefits: while some of the spectral bands require accuracies at (and possibly beyond) the limits of existing technologies, some other specifications could be relaxed. As a specific example for the temperature retrievals, the rate of change of in-band radiance with temperature is very fast in the MWIR, which allows a relaxation of calibration accuracies for those bands.

Establishing laboratory standards for calibrations proved to be a challenging task. We decided that for the desired accuracies we should directly reference our calibrations to the National Institute for Standards and Technology (NIST). This traceability could be established at several levels: we decided that direct comparisons of radiance to the national standards would be most appropriate. (Alternative approaches might include tracing the temperature sensors on blackbodies, or using various transfer standards. However, each of these would engender too much uncertainty for our application.) Our need for direct traceability required us to develop two new sources: a highly accurate vacuumcompatible blackbody [37] and a vacuum-compatible integrating sphere source [38]. These sources have been directly calibrated at NIST (additionally the blackbody, shown in Figure 4, is being adopted by NIST for use in their new Medium Background Infra Red (MBIR) facility). At Los Alamos, we have incorporated these sources, plus others, into our Radiometric Calibration Station, RCS [39] and [40]. Other sources for the RCS include a spectrometer and laser interferometer as well as a variety of targets that can be illuminated by the various sources. All of these sources can be positioned at the focus of a reflective telescope, which generates a 25 -inch diameter collimated output for calibration of MTI and other instruments. The RCS will be used for measurements of the spatial, spectral and radiometric properties of the instrument.

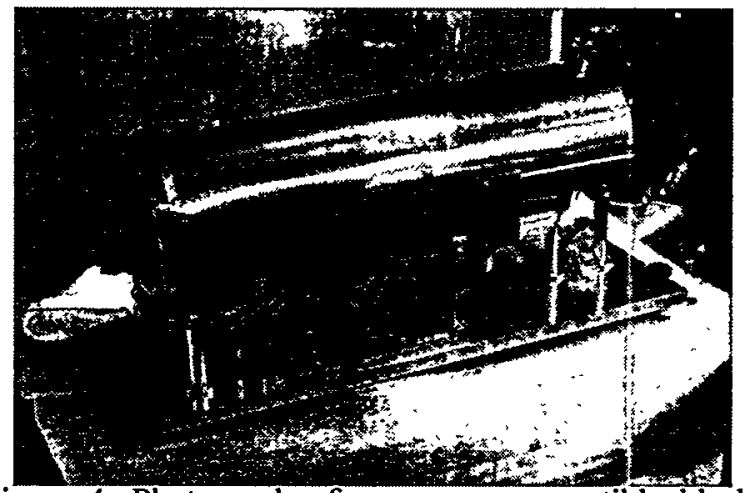

Figure 4: Photograph of a vacuum-compatible blackbody transfer standard. 
The RCS calibration really serves to transfer the NIST standards to the onboard calibration system for MTI. That system (see Figure 5) was specified through the EEM, and includes both a full-aperture calibrator and a "quick-look" calibration wheel with several sources. The full-aperture calibrator is a clamshell structure that also serves as the aperture door for the MTI instrument. One segment of the clamshell is a temperature-controlled blackbody, which is used for the infrared bands. The second segment is a diffuse reflector, which can be oriented to admit sunlight to the system for calibration of the shorter wavelength bands. We can check the reflectivity of the diffuse reflector using a small cavity monitor, which has filtered photodiode detectors. These detectors match the spectral content of the shortest four bands on MTI. Based on experience with other satellite instruments, we anticipate initially performing fullaperture calibrations on approximately a weekly basis. The "quick-look" calibration system is located at the entrance of the dewar, which contains the focal plane arrays, and we plan to use it immediately before and immediately after acquiring each terrestrial observation. This is especially important in view of the $1 / \mathrm{f}$ drifts that are endemic in the infrared detectors. The "quick-look" sources consist of two blackbodies, two lamps, and a highly reflective element on a wheel that allows rapid selection of each source. A more detailed description of the hardware components in the onboard calibration systems is provided by Zalewski et al [41].

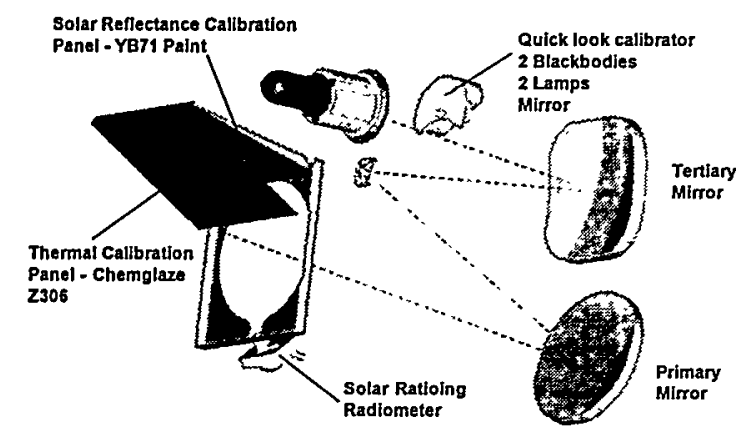

Figure 5: Major elements of the on-board calibration system.

Vicarious calibrations will be conducted when the payload is in orbit. We have selected a number of targets for which ground truth will be available, and we plan to obtain periodic data on these sites over the full operational life of the MTI satellite. In addition to ground sites, we are evaluating the possibility of using airborne sensors, either for direct inter-comparisons, or as intermediate calibration systems between MTI and other satellite payloads (such as the NASA Earth Observing System multispectral sensors) which are in different orbits.

\section{SUMMARY}

We have described an approach to remote sensing in which we use physics-based end-to-end modeling to establish the most appropriate measurement strategies. This approach allows us to develop a fully self-consistent approach to specifications of hardware, calibration, analysis software, and deployment. The approach is modular, and, since it is based in physics, it is scalable to other applications.

We described in general terms the application of these measurement strategies to the Multispectral Thermal Imager satellite payload, and provided references to various more detailed papers. As of the time of this writing, the MTI payload is assembled and under test; it will soon be brought to the Los Alamos calibration facility for detailed calibrations. Launch is scheduled for the end of 1999. The ultimate test of our approach will be the performance of the system during orbital operations, which are slated to last at least fourteen months.

\section{ACKNOWLEDGMENTS}

The US Department of Energy supports this work at Los Alamos National Laboratory, Sandia National Laboratory, and the Savannah River Technology Center. The authors are very grateful to their colleagues who contribute to the work described in this paper, and to the management of their Laboratories for their support.

\section{REFERENCES}

[1] H. S. Chen, Space Remote Sensing Systems, Academic Press, New York 1985.

[2] H. J. Kramer, Observation of the Earth and its Environment, Springer-Verlag, New York, 1994.

[3] David L. Glackin: "Overview of International Remote Sensing through 2007", in Sensors, Systems and NextGeneration Satellites, Hiroyuki Fujisada, Editor, Proceedings of SPIE Vol. 3221, 504-511 (1997).

[4] James E. Slattery and Paul R. Cooley, "Space-Based Infrared Satellite System (SBIRS) Requirements Management" 1998 IEEE Aerospace Applications Conference Proceedings, 223-232, 1998.

[5] Gregg Vane and Alexander F. H. Goetz, "Terrestrial Imaging Spectrometry: Current Status, Future Trends", Remote Sensing of the Environment, 44, 117-12, 1993.

[6] Rick Piltz, Editor, Our Changing Planet, Global Change Research Information Office, University Center, MI, 1998.

[7] Tom Wilson and Robert Felt, "Hyperspectral Remote Sensing Technology (HRST) Program", 1998 IEEE 
Aerospace Applications Conference Proceedings, 193-200, 1998.

[8] John J. Bates, "A Decade of Multispectral Sea Surface Temperature Observations from Space", Advances in Space Research, 14, 5-14, 1994.

[9] A. J. Prata, "Land Surface Temperature Determination from Satellites", Advances in Space Research, 14, 15-26, 1994.

[10] Garik G. Gutman, "Multi-Annual Time Series of AVHRR-Derived Land Surface Temperature", Advances in Space Research, 14, 27-30, 1994.

[11] J. Dozier and Z. Wan: "Development of Practical Multiband Algorithms for Estimating Land Surface Temperature from EOS/MODIS Data", Advances in Space Research, 14, 81-90, 1994.

[12] R. Rex Kay, Brian C. Brock, Tammy D. Henson, Jeffrey L. Rienstra, Max L. Decker, N. Glenn Rackley, Paul G. Weber, Steven C. Bender and Donald A. Byrd, "An introduction to the Department of Energy's Multispectral Thermal Imager Project Emphasizing the Imaging and Calibration Subsystems", Proc. $12^{\text {th }}$ AIAA/USU Conference on Small Satellites, Utah State University, September 1998.

[13] Zhengming Wan, Wil Snyder and Yulin Zhang, "Validation of Land-Surface Temperature Retrieval from Space", Proceedings of the 1996 IEEE International Geoscience and Remote Sensing Symposium, Lincoln, Nebraska, IEEE Catalog number 96CH35875, 1996.

[14] A. J. Garrett, SRTC, Personal Communication, 1998.

[15] A. J. Garrett and D. W. Hayes, "Cooling Lake Simulations Compared to Thermal Imagery and Dye Tracers." J. Hydr. Engineering., 123(10), 885-894, 1997.

[16] C. Cox and W. Munk, "Measurement of the Roughness of the Sea Surface from Photographs of the Sun's Glitter", J. Opt. Soc. Am. Vol. 44, 838-850, 1954.

[17] James Theiler and Bradley G. Henderson, "A Geometrical Constraint on Shadowing in Rough Surfaces", Infrared Spaceborne Remote Sensing V, Marija Strojnik and Bjorn Andresen, Editors, Proceedings of SPIE Vol. 3122, 271-279, 1997.

[18] Bradley G. Henderson, James Theiler and Pierre V. Villeneuve, "The Polarized Emissivity of a WindRoughened Sea Surface: A Monte Carlo Model", Remote Sensing of the Environment, 1997.

[19] J. R. Schott, B. V. Brower, R. Bashkar, R. Raqueno and C. Salvaggio, technical Report RIT/DIRS 90/91-51-140,
Digital Imaging and Remote Sensing Laboratory, Rochester Institute of Technology 1991.

[20] Graeme L. Stephens, Remote Sensing of the Lower Atmosphere, Oxford University Press, New York, 1994.

[21] R. M. Goody and Y. L. Yung, Atmospheric Radiation Theoretical Basis, Oxford University Press, New York, 1989.

[22] Moira L. Steyn-Ross and D. Alistair Steyn-Ross, "Land Surface Temperature Retrieval from AVHRR: Influence of Surface Emissivity and Atmospheric Water Vapor", Proceedings of the 1996 IEEE International Geoscience and Remote Sensing Symposium, Lincoln, Nebraska, IEEE Catalog number 96CH35875, 2098-2100, 1996.

[23] T. J. Schmugge and G. M. Schmidt, "TIR Observations in FIFE from Field, Aircraft and Satellite Platforms", Proceedings of the 1996 IEEE International Geoscience and Remote Sensing Symposium, Lincoln, Nebraska, IEEE Catalog number 96CH35875, 2101-2103, 1996.

[24] Christopher S. Ruf, "Pathfinder SSM/I Data" in IEEE Geoscience and Remote Sensing Society Newsletter, September 1997.

[25] Daniel Schlapfer, Christoph C. Borel, Johannes Keller and Klaus I. Itten, "Atmospheric Precorrected Differential Absorption Technique to Retrieve Columnar Water Vapor", Remote Sensing of the Environment, 65, 353-366, 1998.

[26] A. H. Goodman and A. Henderson-Sellers, "Cloud Detection Analysis - A Review of Recent Progress", Atmospheric Research, 21, 203, 1988.

[27] W. B. Rossow and L. C. Garder, "Cloud Detection Using Satellite Measurements of Infrared and Visible Radiances for ISCCP", J. Climate 6, 2341-2369, 1993.

[28] B. -C. Gao, A. F. H. Goetz and W. J. Wiscombe, "Cirrus Cloud Detection from Airborne Imaging Spectrometer Data Using the 1.38 um Water Vapor Band", Geophys. Res. Letters, 20, 301-304, 1993.

[29] Bo-Cai Gao, Curtiss O. Davis and Yoram J. Kaufman, "Thin Cirrus Detection and Correction of Thin Cirrus Path Radiances Using near-IR Channels Near 1.375 um", Infrared Spaceborne Remote Sensing V, Marija Strojnik and Bjorn Andresen, Editors, Proceedings of SPIE Vol. 3122, 78-87, 1997.

[30] Bradly J. Cooke, Bryan E, Laubscher, Christoph C. Borel, Terrence S. Lomheim and Christopher F. Klein, "Methodology for Rapid Infrared Multi-Spectral, ElectroOptical Imaging System Performance Analysis and Synthesis", Aerospace / Defense Sensing and Controls, Proceedings of SPIE, 8-12 April 1996. 
[31] Jeffrey Rienstra and Mary Ballard, "Multispectral Focal Plane Assembly for Satellite Remote Sensing", 1998 IEEE Aerospace Applications Conference Proceedings, 233-241, 1998.

[32] W. B. Clodius, P.G. Weber, C. C. Borel and B. W. Smith, "Multi-spectral band selection for satellite-based systems", SPIE Conference on Imaging Systems: design, Modeling and testing IX, 15-16 April 1998, Orlando, Florida, to be published in Proc. SPIE Vol. 3377, paper 3377-02 (1998).

[33] David A. Bearden, Donald P. Duclos, Mark J. Barrera, Todd J. Mosher and Norman Y. Lao, "A Methodology for Conceptual Remote Sensing Spacecraft Technology Insertion Analysis Balancing Performance, Cost and Risk", Sensors, Systems and Next-Generation Satellites, Hiroyuki Fujisada, Editor, Proceedings of SPIE Vol. 3221, 536-549 (1997).

[34] James R. Wertz and Wiley J. Larson, Editors, Space Mission Analysis and Design, Kluwer Academic Publishers, Boston, 1991.

[35] James Theiler, Bradley G. Henderson and Barham W. Smith, "Algorithms Using Inter-band Cross-correlation for Pixel Registration and Jitter Reconstruction in Multichannel Push-broom Imagers", Proceedings of SPIE Vol. 3163 (1997).

[36] Christoph C. Borel and Siegfried A. W. Gerstl, "Accurate Top of the Atmosphere Albedo Determination from Multiple Views of the MISR Instrument", Proceedings of the 1996 IEEE International Geoscience and Remote Sensing Symposium, Lincoln, Nebraska, IEEE Catalog number 96CH35875, 1996.

[37] D. A. Byrd, F. D. Michaud, S. C. Bender, et al, "Design, Manufacture, and Calibration of Infrared radiometric Blackbody Sources", Aerospace / Defense Sensing and Controls, Proceedings of SPIE, 8-12 April 1996.

[38] D. A. Byrd, W. H. Atkins, S. C. Bender, R. W. Christensen and F. D. Michaud, "Vacuum-Compatible Standard Diffuse Source: Manufacture and Calibration" in Illumination and Source Engineering, Proceedings of SPIE, Vol. 3428, 9-22 (1998).

[39] S. C. Bender, W. B. Maier II, D. Byrd, et al, "The Los Alamos Calibration Laboratory for Multi-spectral and Thermal Imaging Radiometer Systems", Proceedings Fourth SDLUSU Symposium on Infrared Radiometric Sensor Calibration, 1994.

[40] D. A. Byrd, W. B. Maier II, S. C. Bender, et al: "Visible / Infrared Radiometric Calibration Station", Proceedings of the SPIE, Vol. 2268, 398-403, 1994.
[41] E. F. Zalewski, W. M. Rapoport, F. R. Sileo, et al, "Onboard Calibration System for a Visible-to-Thermal Infrared Multispectral Imaging Sensor", Proceedings of the SPIE, Vol. 3439, paper 47, 1998

\section{BIOGRAPHY}

Paul Weber earned a Ph.D. in Physics from the Flinders University of South Australia in 1976. He then accepted a research position at Columbia University, where he worked on the equilibrium and stability of plasmas at high pressures. Since 1980 he has been at Los Alamos, starting in the Advanced Diagnostics Group, where he developed several particle beam, laser, and spectroscopic systems. In 1986 he assumed responsibility for experimental Reversed Field Pinch (RFP) physics within the magnetic confinement fusion program, concentrating personally on impurity dynamics and plasma confinement. In 1991 he moved to Space Science and Technology Division, where he pursued several new initiatives in (mostly) optical and infrared remote sensing for nonproliferation, energy production, pollution, environment, and global change. In subsequent years he became the Los Alamos Project Leader and Principal Investigator for several large projects. In 1993 he was selected as a Deputy Group Leader of the Astrophysics and Radiation measurements Group. In 1997 he became Group Leader of the Space and Remote Sensing Sciences Group. Paul Weber is the author, or co-author, of over 160 publications, conference papers and reports. 